\title{
Plastia cirúrgica de óstio coronariano esquerdo com estenose não aterosclerótica
}

\author{
Fábio ARGENTA*, George WINCKLER* ${ }^{*}$ Marcelo Kuhn MOMOLLI*, Roque FALLEIRO*, \\ Luís Sérgio FRAGOMENI*
}

RBCCV 44205-521

\begin{abstract}
Argenta F, Winckler G, Momolli M K, Falleiro R, Fragomeni L S - Plastia cirúrgica de óstio coronariano esquerdo com estenose não aterosclerótica. Rev Bras Cir Cardiovasc 2000; 15(4): 345-8.
\end{abstract}

RESUMO: Discute-se o caso clínico de um paciente homem, 38 anos, com insuficiência cardíaca classe IV (NYHA) e angina de repouso em razão de insuficiência aórtica severa, oclusão de óstio da artéria coronária direita e lesão subtotal do óstio da artéria coronária esquerda, em razão de aortite de origem luética. $O$ tratamento constituiu-se de prótese aórtica mecânica e plastia do óstio coronariano esquerdo com "telhado" de veia safena. A evolução clínica pós-operatória foi excelente, tendo o paciente retornado à vida ativa e sem sintomas.

DESCRITORES: Artérias coronárias, cirurgia. Aorta, insuficiência. Revascularização miocárdica, cirurgia. Valva aórtica, prótese. Valva aórtica, cirurgia. Implante de prótese de valva.

\section{INTRODUÇÃO}

A incidência de estenose de óstio coronariano (EOC) em duas grandes séries de mais de quatro mil pacientes submetidos a angiografia coronariana foi de $0,13 \%$ e $0,25 \%{ }^{(1,2)}$. Em dois outros estudos, EOC isolada foi encontrada, em somente $0,07 \%$ e $0,09 \%$ dos mais de sete mil pacientes submetidos a coronariografia $(3,4)$. Estas lesões têm sido mais freqüentemente causadas por doença aterosclerótica da aorta, confirmadas por autópsia e exame anatomopatológico ${ }^{(3,5)}$. Entre as causas menos comuns de EOC, podem-se citar a aortite luética, a arterite de Takayasu, a displasia fibromuscular, a doença da valva aórtica, a hipercolesterolemia familiar e a doença congênita da aorta $(1,3,5,6)$.
A técnica de correção cirúrgica usual dessas lesões é o bypass coronariano, usando-se enxertos arteriais como artéria torácica interna (ATI), radial e gastroepiplóica ou a veia safena (VS). Entretanto, a plastia cirúrgica do óstio coronariano pode ser, em casos selecionados, a alternativa ideal para restabelecer o fluxo coronariano em pacientes acometidos de lesão única no óstio coronariano $(6,7)$.

Descreve-se, aqui, o caso de um paciente com aortite de origem luética com oclusão do óstio da artéria coronária direita e com lesão subtotal do óstio da artéria coronária esquerda. O quadro clínico era exacerbado por insuficiência aórtica severa e grave disfunção ventricular. O tratamento cirúrgico adotado foi o implante de prótese valvular aórtica

\footnotetext{
Trabalho realizado no Hospital São Vicente de Paulo - Faculdade de Medicina da Universidade de Passo Fundo. Passo Fundo, RS, Brasil. Recebido para publicação em novembro de 1999.

* Do Hospital São Vicente de Paulo.

Endereço para correspondência: Luís Sérgio Fragomeni. Rua Teixeira Soares, 777, sl. 702. Passo Fundo, RS, Brasil. CEP 99010-080. Tel. (54) 3116762. Fax. (54) 311-1423. e-mail: fragomeni@annex.com.br
} 
Argenta F, Winckler G, Momolli M K, Falleiro R, Fragomeni L S - Plastia cirúrgica de óstio coronariano esquerdo com estenose não aterosclerótica. Rev Bras Cir Cardiovasc 2000; 15(4): 345-8.

e a ampliação do tronco da artéria coronária esquerda com um enxerto de veia safena como alternativa ao bypass tradicional.

\section{CASUÍSTICA E MÉTODOS}

\section{Relato do Caso}

Homem, branco, 38 anos, com angina de repouso após infarto agudo do miocárdio recente e insuficiência cardíaca classe IV (NYHA). No passado, teve quadro clínico de Luess tratado. Cineangiocoronariografia evidenciou insuficiência aórtica severa, oclusão do óstio coronariano direito e lesão subtotal do óstio coronariano esquerdo. Com exceção das lesões citadas, as artérias coronárias eram morfologicamente normais. A fração de ejeção do ventrículo esquerdo era de 0,30 (Figura 1). A abordagem cirúrgica foi através de esternotomia mediana com auxílio de circulação extracorpórea (CEC), hipotermia sistêmica moderada e proteção miocárdia com hipotermia tópica e solução cardioplégica anterógrada no óstio coronariano esquerdo. A aorta ascendente tinha seu diâmetro preservado, mas as alterações macro e microscópicas das camadas da aorta eram típicas de aortite. A valva aórtica apresentava-se francamente insuficiente em razão de absorção central dos seus foIhetos. Após a remoção dos folhetos da valva aórtica, foi implantada uma prótese aórtica metálica de disco pivotante. A seguir, a lesão ostial esquerda foi abordada após a incisão da aorta ter sido estendida até o óstio coronariano e ampliada por mais dois centímetros. Para que esse acesso fosse possível, o tronco da artéria pulmonar foi seccionado transversalmente. Com um segmento de VS, realizou-se um "telhado" no tronco esquerdo até a aorta, de forma a ampliar adequadamente o óstio estenosado. A incisão da aorta foi fechada com sutura contínua e a artéria pulmonar foi reconstituída por uma anastomose término-terminal. Após a saída da CEC, o sangramento da região posterior da aorta era abundante e, para corrigí-lo, houve necessidade de novo pinçamento aórtico. O alargamento realizado no tronco da artéria coronária esquerda foi ressuturado num local de rotura onde a parede do vaso era mais rígida em razão da calcificação. O tempo de pinçamento aórtico foi de 120 minutos.

\section{RESULTADOS}

O paciente teve evolução pós-operatória satisfatória, com alta hospitalar em boas condições clínicas 14 dias após o ato cirúrgico. A evolução,

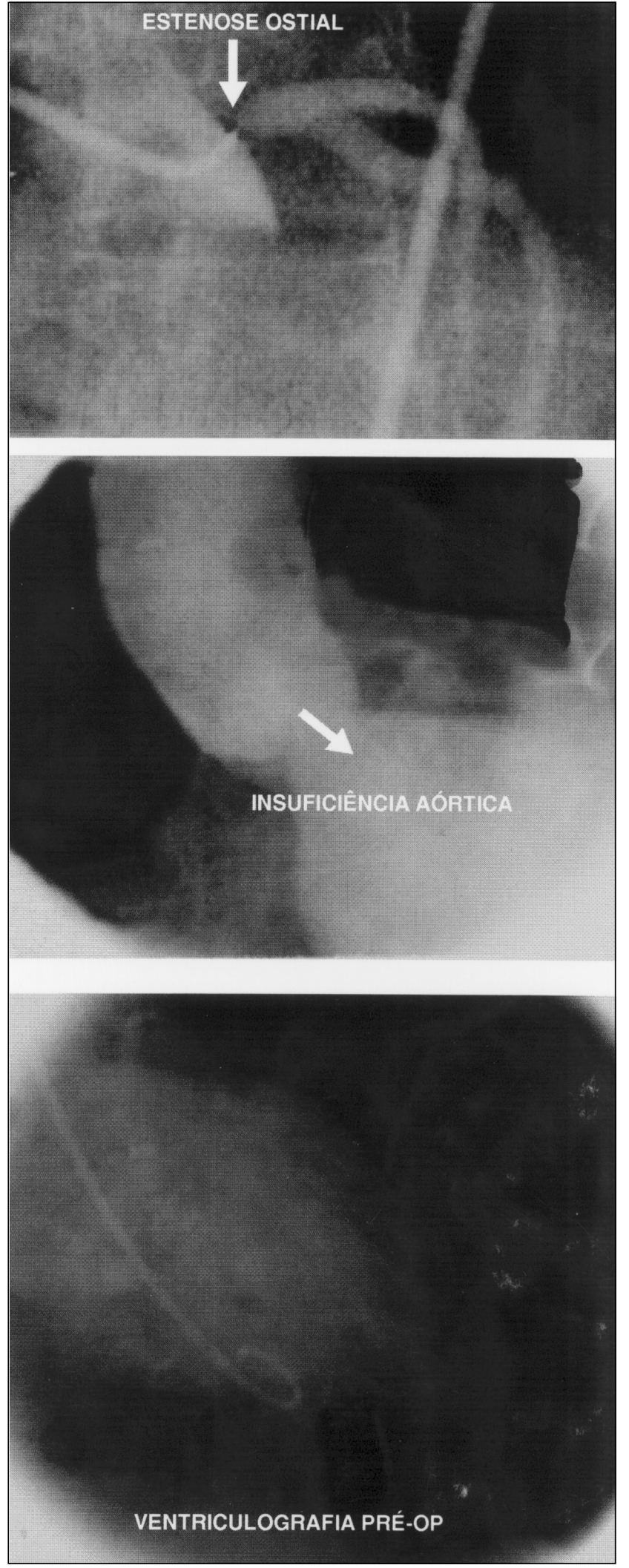

Fig. 1 - Avaliação pré-operatória, onde se identificam a lesão do óstio da artéria coronária esquerda, a insuficiência aórtica e disfunção severa do ventrículo esquerdo. 
Argenta F, Winckler G, Momolli M K, Falleiro R, Fragomeni L S - Plastia cirúrgica de óstio coronariano esquerdo com estenose não aterosclerótica. Rev Bras Cir Cardiovasc 2000; 15(4): 345-8.

com um seguimento de 52 meses, revela que o paciente reassumiu suas atividades normais, livre de angina, encontrando-se em classe funcional I. O reestudo hemodinâmico, realizado após quatro meses, demonstrou ampla abertura do óstio coronariano esquerdo e também melhora significativa na função ventricular (Figura 2).

\section{COMENTÁRIOS}

A abordagem cirúrgica para reparar a EOC foi pioneira por DUBOST et al. ${ }^{(8)}$, em 1960, e por CONNOLLY et al. (9), em 1964, tendo ambos utilizado endarterectomia transaórtica para reparar obstruções ostiais sifilíticas. Em 1965, EFFLER et al. ${ }^{(10)}$ e SABISTON et al. (11) foram os primeiros a relatar uma série de ostioplastias, porém esses procedimentos foram posteriormente abandonados, em virtude das altas taxas de mortalidade (acima de $45 \%$ ). Entretanto, em 1983, com o advento da melhor proteção miocárdica, HITCHCOCK et al. (12), reviveram com sucesso os conceitos da ostioplastia e seus excelentes resultados encorajaram outros cirurgiões a utilizar essa técnica em grupos de pacientes selecionados. Subseqüentes relatos por SULLIVAN \& MURPHY (13) e por DION et al. (14) confirmaram a utilidade desse procedimento.

A técnica de ostioplastia utilizando-se enxertos de VS ou pericárdio bovino pode ser empregada para restaurar um fluxo sangüíneo adequado tanto em estenoses ostiais da artéria coronária direita como da esquerda. Preferencialmente, a plastia cirúrgica do óstio deve ser aplicada em pacientes com lesão ostial única, como no caso exposto, porém há casos já descritos em que a ostioplastia foi associada ao bypass convencional, com resultados satisfatórios (7). Sabe-se que pacientes com idade avançada, lesões calcificadas e envolvimento de bifurcações distais contra-indicam essa técnica. Embora aplicada apenas a um número selecionado de pacientes com doença isquêmica cardíaca, citam-se como vantagens a restauração da anatomia normal coronariana, uma circulação sangüínea anterógrada mais fisiológica ratificada pelos princípios de Prizometer ${ }^{(14)}$ e a preservação do material venoso ou arterial que seria usado em possíveis intervenções futuras.

\section{CONCLUSÃO}

A reconstrução do óstio coronariano por abordagem direta, especialmente quando for 0 esquerdo, através de retalho autólogo, é uma ótima alternativa para se restabelecer o fluxo

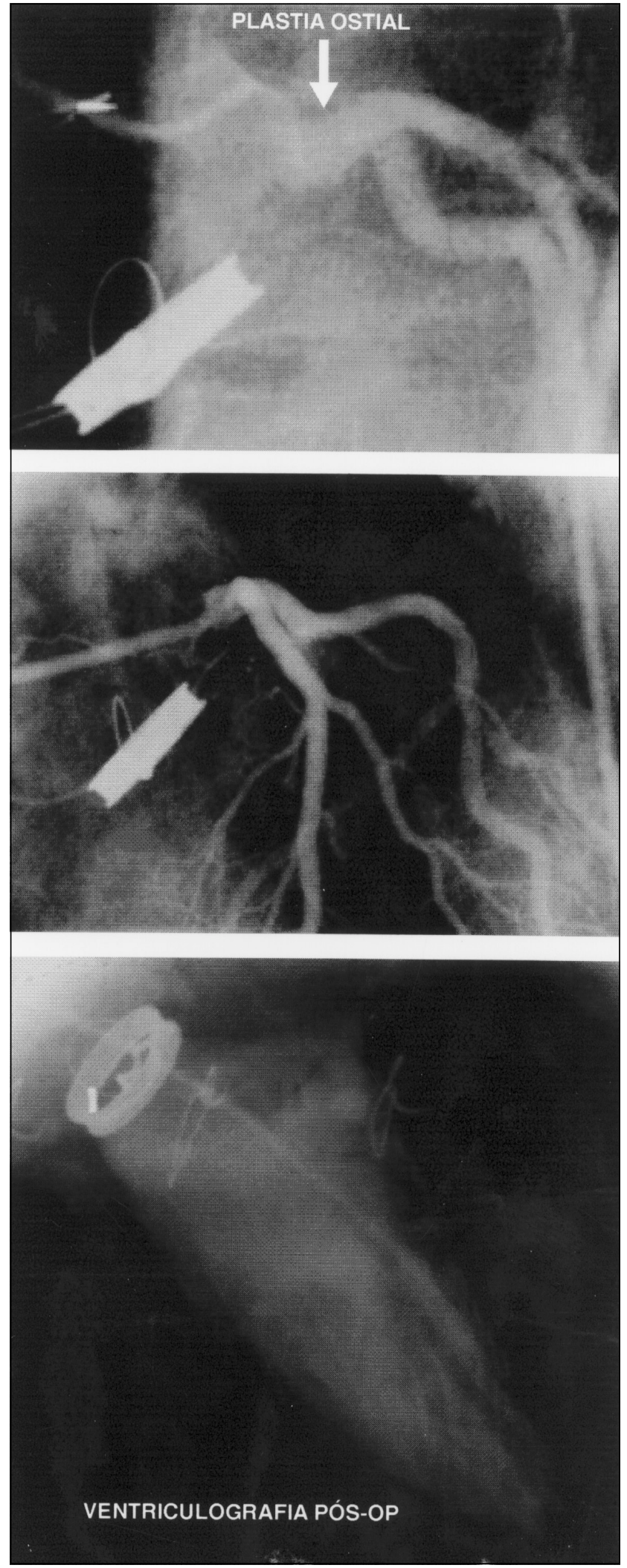

Fig. 2- Controle pós-operatório aos 4 meses, demonstrando-se o resultado satisfatório da plastia do óstio coronariano esquerdo e a recuperação da função ventricular esquerda. 
coronariano normal em pacientes com estenoses ostiais isoladas. Entretanto, por ser uma técnica cirúrgica mais elaborada, em que o índice de complicações, especialmente sangramento, é mais crítico, deve ser empregada com cautela. Cada caso deve ser julgado em particular e, após criteriosa avaliação pré-operatória, a técnica cirúrgica mais apropriada escolhida. Caso se opte pelo bypass tradicional, as ATIs são uma ótima alternativa, seguido dos demais enxertos arteriais, hoje tidos como preferenciais sobre os enxertos venosos. 0 importante é que o cirurgião escolha a opção em que se sinta mais confiante, e que a obstrução coronariana e todas as lesões associadas sejam abordadas simultaneamente.

RBCCV 44205-521

Argenta F, Winckler G, Momolli M K, Falleiro R, Fragomeni L S - Surgical ostial angioplasty of non-atherosclerotic left coronary artery stenosis. Rev Bras Cir Cardiovasc 2000; 15(4): 345-8.

ABSTRACT: A clinical case of a 38 year old male, with heart failure grade IV (NYHA) and angina at rest related to severe aortic regurgitation, right coronary ostial occlusion, and a sub-total occlusion of the left coronary ostium owing to syphilitic infection in the past is reported and discussed. Treatment was established implanting a mechanical aortic prosthesis and a direct left main angioplasty suturing a patch made of safenous vein. Follow up has been excellent and the patient is today having a normal life, free of symptoms.

DESCRIPTORS: Coronary arteries, surgery. Aorta, insufficiency, Myocardial revascularization, surgery. Aortic valve prosthesis. Aortic valve, surgery. Heart valve prosthesis, implantation. Aortic valve stenosis, surgery.

\section{REFERÊNCIAS BIBLIOGRÁFICAS}

1 Barner H B, Naunheim K S, Kanter K R et al. - Coronary ostial stenosis. Eur J Cardiothorac Surg 1988; 2: 106-12.

2 Fruend T, Missri J, Landry A - Isolated left main coronary ostial stenosis. Cardiovasc Rev Rep 1987; 72-3.

3 Eng J, Beton D C, Lawson R A, Moussalli H, Nair U R, Rahman A N - Coronary ostial stenosis: surgical considerations. Int J Cardiol 1991; 30: 285-8.

Hutter J A, Pasaoglu I, Williams B T - The incidence and management of coronary ostial stenosis. J Cardiovasc Surg 1985; 26: 581-4.

5 Thompson R - Isolated coronary ostial stenosis in women. J Am Coll Cardiol 1986; 7: 997-1003.

6 Ghosh P K - Coronary ostial reconstruction: technical issues. Ann Thorac Surg 1991; 51: 673-5.

7 Briffa N P, Clarke S, Kugan G, Coulden R, Wallwork J, Nashef S A - Surgical angioplasty of the left main coronary artery: follow-up with magnetic resonance imaging. Ann Thorac Surg 1996; 62: 550-2.

8 Dubost C, Blondeau P, Piwnica A et al. - Syphilitic coronary obstruction: correction under artificial heartlung and profound hypothermia at $10^{\circ} \mathrm{C}$. Surgery 1960; 48: 540-7.
9 Connolly J E, Eldridge F L, Calvin J W, Stemmer E A - Proximal coronary-artery obstruction: its etiology and treatment by transaortic endarterectomy. $N$ Engl J Med 1964; 271: 213-9.

10 Effler D B, Sones F M Jr., Favoloro R, Groves L K - Coronary endarterectomy with patch-graft reconstruction: clinical experience with 34 cases. Ann Surg 1965; 162: 590-601.

11 Sabiston D C Jr., Ebert P A, Friesinger G C Ross R S, Sinclair Smith B - Proximal endarterectomy: arterial reconstruction for coronary occlusion at aortic origin. Arch Surg 1965; 91: 758-64.

12 Hitchcock J F, Robles de Medina E O, Jambroes $G$ - Angioplasty of the left main coronary artery for isolated left main coronary artery disease. J Thorac Cardiovasc Surg 1983; 85: $880-4$.

13 Sullivan J \& Murphy D A - Surgical repair of stenotic ostial lesions of the left main coronary artery. $J$ Thorac Cardiovasc Surg 1989; 98: 33-6.

14 Dion R, Verhelst R, Matta A, Rousseau M, Goenen M, Chalant C - Surgical angioplasty of the left main coronary artery. J Thorac Cardiovasc Surg 1990; 99: $241-50$. 